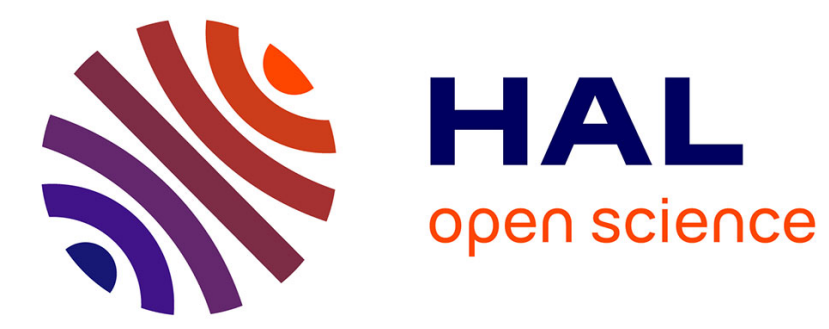

\title{
Multiplication in vitro du noisetier (Corylus avellana L.)
}

Hanan Al Kaï, Georges Salesses, Armand Mouras

\section{To cite this version:}

Hanan Al Kaï, Georges Salesses, Armand Mouras. Multiplication in vitro du noisetier (Corylus avellana L.). Agronomie, 1984, 4 (4), pp.399-402. hal-00884651

\section{HAL Id: hal-00884651 https://hal.science/hal-00884651}

Submitted on 1 Jan 1984

HAL is a multi-disciplinary open access archive for the deposit and dissemination of scientific research documents, whether they are published or not. The documents may come from teaching and research institutions in France or abroad, or from public or private research centers.
L'archive ouverte pluridisciplinaire HAL, est destinée au dépôt et à la diffusion de documents scientifiques de niveau recherche, publiés ou non, émanant des établissements d'enseignement et de recherche français ou étrangers, des laboratoires publics ou privés. 


\title{
Multiplication in vitro du noisetier (Corylus avellana L.)
}

\author{
Hanan AL KAÏ, Georges SALESSES \& Armand MOURAS $\left({ }^{*}\right)$ \\ I.N.R.A., Station d'Arboriculture fruitière, Centre de Recherches de Bordeaux F 33140 Pont-de-la-Maye \\ (*) Laboratoire de Biologie cellulaire, Université de Bordeaux II, F 33400 Talence
}

RÉSUMÉ

\begin{abstract}
La micropropagation, si elle permet la mise à disposition rapide des nouveaux cultivars issus des programmes d'amélioration, deviendra un facteur important pour l'évolution du verger de noisetier.

Sur matériel jeune, il a été possible d'obtenir, à partir de bourgeons axillaires, un taux de multiplication intéressant, en apportant le fer sous forme d'un chélate du commerce peu utilisé en culture in vitro ; l'enracinement et le transfert des plants sur substrat normal ne soulèvent pas de problème particulier.

Malgré les résultats encourageants obtenus avec matériel adulte, des améliorations doivent encore être apportées à la technique, avant d'envisager une utilisation intensive.
\end{abstract}

Mots clés additionnels : Micropropagation, multiplication végétative, culture in vitro.

In vitro multiplication of hazel (Corylus avellana $L$.).

\begin{abstract}
If new cultivars of hazel are to be distributed more rapidly than through horticultural methods, in vitro propagation will have to be considered as an important factor in future developments. From young material, it was possible to obtain a good multiplication rate without special problems for rooting or establishment in soil. To promote multiplication of axillary buds ; best results were obtained with Murashige and Skoog basal medium supplemented with Zuccherelli vitamins, NAA, GA and benzylaminopurine at concentrations 0,01 , 0.1 and $5 \mathrm{mg} / \mathrm{l}$. The nature of the iron supply seemed to be very important : thus Fe EDTA gave poor results with very chlorotic shoots, while Sequestrene $138 \mathrm{Fe}$ (Ciba-Geigy) gave better results. From adult material, some fairly good results were obtained with the same culture medium but the method has to be improved for an intensive use.
\end{abstract}

Additional key words : Micropropagation, vegetative propagation, in vitro culture.

\section{INTRODUCTION}

Après sélection ou création d'une nouvelle variété de noisetier se pose le problème de sa multiplication et de sa mise à disposition rapide. L'établissement d'une touffe nécessitant environ 4 années avant qu'elle puisse être multipliée par les techniques horticoles habituellement utilisées (marcottage en crosse ou par étranglement de rejets), la multiplication d'un cultivar nouveau peut demander 8 à 10 années. Ces faits constitueront certainement un facteur limitant pour l'évolution variétale que l'on est en mesure d'attendre après les importants travaux de sélection et de création réalisés ces dernières années.

La multiplication in vitro doit pouvoir constituer une méthode plus rapide de production de plants, qui conduira vraisemblablement aussi à un abaissement du coût de production. La mise au point d'une technique de micropropagation peut donc se révéler importante pour l'évolution du verger de noisetier.
En outre, les vitroplants peuvent constituer un matériel de choix pour aborder de façon plus efficace certaines études de physiologie, de pathologie ou de génétique.

Si des résultats intéressants ont été obtenus sur d'autres espèces fruitières (QUOIRIN et al., 19761977 ; SEIRLIS et al., 1979 ; ZUCCHERELLI, 1979 ; CHEVRE et al., 1983), à notre connaissance, les seuls travaux réalisés chez le noisetier jusqu'à ce jour portent sur la culture d'embryons immatures (RADOJEVIC, 1980) et sur celle de cotylédons (PEREZ et al., $1982)$; il en est résulté une embryogenèse somatique dans le premier cas, la formation de cals et de racines dans le second.

Nous ferons état en ce qui nous concerne des premiers résultats obtenus quant à la micropropagation à partir de bourgeons axillaires, technique qui, a priori, doit conduire à la multiplication conforme d'un matériel choisi. 


\section{MATÉRIEL ET MÉTHODES}

Trois variétés ont été utilisées : «Segorbe », «Fertile de Coutard» et «Merveille de Bollwiller ».

Les tentatives ont été réalisées sur matériel juvénile, constitué de plantules de 2 mois issues de semis, et de matériel plus âgé provenant soit de pieds mères pour marcottage soit de marcottes de 2 ans cultivées en serre ; ce $2^{\text {e }}$ type de matériel sera qualifié d'adulte par opposition au matériel juvénile. Dans les 2 cas, les explantats sont prélevés sur la partie non lignifiée de tiges en croissance.

Deux méthodes de stérilisation ont été appliquées :

\section{A. Matériel juvénile}

- passage dans l'alcool à $70^{\circ}$, pendant 30 à $60 \mathrm{~s}$;

- trempage dans une solution d'hypochlorite de sodium à 14 p. 100 additionnée de quelques gouttes d'un agent mouillant (Tween 20) pendant $15 \mathrm{mn}$;

- trois rinçages successifs à l'eau distillée stérile.

\section{B. Matériel adulte}

- passage dans l'alcool à $70^{\circ}$, pendant 30 à $60 \mathrm{~s}$;

- trempage pendant $10 \mathrm{mn}$ dans une solution de chlorure mercurique à $1 \mathrm{~g} / \mathrm{l}$ additionnée de quelques gouttes de Tween 20 ;

- rinçage dans une solution de chlorure de calcium à $2,5 \mathrm{~g} / 1$ afin de limiter la toxicité due au mercure ;

- trois rinçages à l'eau distillée stérile.

Si le taux d'infection est relativement bas sur matériel jeune, il reste très variable et toujours supérieur sur matériel adulte.

Les explantats, comprenant un bourgeon axillaire et une portion de tige de 1 à $2 \mathrm{~cm}$, sont mis en culture sur des milieux solidifiés par la gélose à $7 \mathrm{~g} / \mathrm{l}$ dans des tubes placés dans une salle dont la température est maintenue à $25 \pm 1{ }^{\circ} \mathrm{C}$ avec une photopériode de $16 \mathrm{~h}$ de lumière (2000 lux) et $8 \mathrm{~h}$ d'obscurité.

Les divers milieux utilisés comprennent :

- La solution minérale de QUOIRIN et al. (197677) ou celle de MuraSHige \& SKOOG (1962).

Le fer apporté dans ces solutions sous forme de $\mathrm{Fe}$ EDTA est remplacé pour certaines phases par le Séquestrène $\mathrm{Fe} 138$ (EDDHA) Ciba-Geigy ${ }^{\circledR}$.

- Les constituants organiques utilisés par MURASHIGE \& SKOOG (1962) ou par ZUCCHERELLI (1979), modifiés quant aux facteurs de croissance par l'apport d'acide indolbutyrique (AIB), d'acide naphtalène acétique (ANA), de benzylaminopurine (BAP), d'adénine (Ad) et d'acide gibbérellique (AG).

Avant stérilisation à l'autoclave à $115^{\circ} \mathrm{C}$ pendant $20 \mathrm{mn}$, le pH des milieux est ajusté à 5,5 .

En ce qui concerne plus spécialement le matériel adulte, les explantats herbacés sont placés sur divers milieux de culture présentés dans le tableau 1.

Les vitroplarts, à la sortie des tubes, sont transférés en serre à $25 \pm 2{ }^{\circ} \mathrm{C}$ sur terreau stérilisé et recouverts d'un capuchon polyéthylène pour maintenir une humidité relative assez importante pendant quelques jours.
TABLEAU 1

Milieux de culture et substances de croissance utilisés sur matériel adulte.

Culture media and growth substances used with adult material.

\begin{tabular}{ccccc}
\hline $\begin{array}{c}\text { Milieux } \\
\text { de base }\end{array}$ & $\begin{array}{c}\text { Mélange } \\
\text { vitaminique }\end{array}$ & $\begin{array}{c}\mathrm{BAP} \\
(\mathrm{mg} / \mathrm{l})\end{array}$ & $\begin{array}{c}\mathrm{AIB} \\
(\mathrm{mg} / \mathrm{l})\end{array}$ & $\begin{array}{c}\mathrm{AG} \\
(\mathrm{mg} / \mathrm{l})\end{array}$ \\
\hline$\frac{\text { M.S. }}{2}$ & M.S. & 5 & - & - \\
\hline M.S. & M.S. & 5 & - & - \\
\hline M.S. & M.S. & 5 & 0,01 & - \\
\hline M.S. & M.S. & 50 & 1 & 1 \\
\hline M.S. & ZuCCH. & 5 & 0,01 & - \\
\hline \hline
\end{tabular}

\section{RÉSULTATS}

\section{A. Matériel juvénile}

Macro et microéléments de MuRASHIGE \& SKOOG d'une part, de QUOIRIN et al. d'autre part, ont été utilisés dans les premiers essais en combinaison avec le mélange vitaminique de MURASHIGE et SKOOG. Les meilleurs résultats ont été notés avec la $2^{\mathrm{e}}$ formule (QUOIRIN et al.) qui a donc été retenue pour la suite du travail.

A cette solution sont additionnés des facteurs de croissance, seuls ou en combinaison : AIB $(0,1$ à $1 \mathrm{mg} / \mathrm{l})$, AG $(0,1$ à $5 \mathrm{mg} / \mathrm{l})$, BAP $(0$ à $50 \mathrm{mg} / \mathrm{l})$ et $\mathrm{Ad}$ $(5 \mathrm{mg} / \mathrm{l})$.

Le démarrage des axillaires se produit de façon plus ou moins régulière avec tous les équilibres testés, mais le meilleur résultat est noté avec le milieu renfermant seulement la BAP à la concentration de $5 \mathrm{mg} / \mathrm{l}$.

\section{Multiplication}

Cette même formule conduit à une multiplication variable avec les bourgeons et les variétés, la meilleure performance étant obtenue avec la variété "Segorbe ". Il est bon de souligner que le déclenchement de la multiplication ne se produit dans certains cas qu'au cours du $2^{\mathrm{e}}$ ou $3^{\mathrm{e}}$ passage : ainsi nous avons obtenu 75 p. 100 de bourgeons en multiplication pour la variété «Segorbe", 70 p. 100 pour «Fertile de Coutard» et 50 p. 100 pour «Merveille de Bollwiller ». Dans tous les cas, la multiplication reste variable et de faible intensité.

Pour améliorer son taux, d'autres milieux de culture ont été essayés ; le meilleur résultat est obtenu avec la formule suivante : macro et microéléments de MURASHIGE \& SKOOG (1962), mélange vitaminique utilisé par ZUCCHERELLI (1979), ANA $(0,01 \mathrm{mg} / \mathrm{l})$, AG $(0,1 \mathrm{mg} / \mathrm{l})$, BAP $(5 \mathrm{mg} / \mathrm{l})$. Après 5 passages sur ce milieu, 1 bourgeon axillaire peut fournir, en moyenne, 20 pousses feuillées (fig. 1).

Au cours de cette phase, nous avons observé, dans tous les cas, une décoloration du limbe foliaire pouvant traduire soit une chlorose soit une autre carence. Des analyses comparatives entre feuilles de pousses issues de la culture in vitro et feuilles d'arbres cultivés en verger n'ont pas permis de faire un diagnostic. 


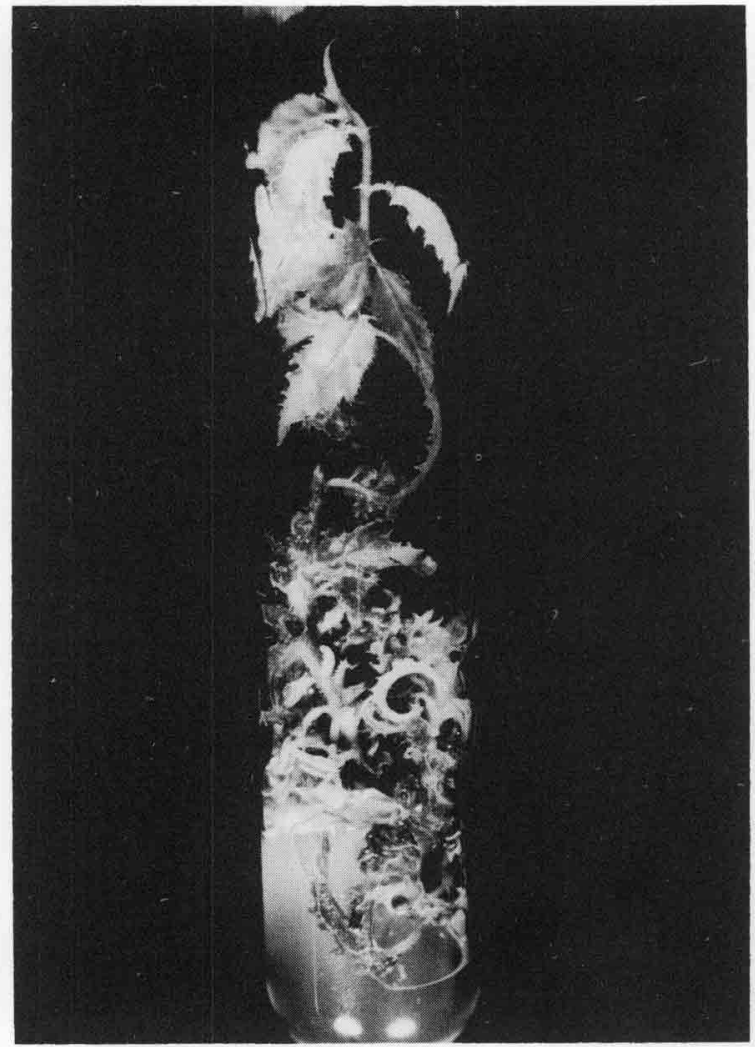

Figure 1

Multiplication obtenue avec matériel juvénile au $5^{e}$ repiquage. Multiplication obtained from juvenile material after 5 subcultures.

Cependant nous avons tenté d'agir de deux façons : en modifiant le $\mathrm{pH}(\mathrm{pH}=6$ au lieu de $\mathrm{pH}=5,5)$ ou l'apport de fer. Si la variation de $\mathrm{pH}$ et le doublement de la dose de fer, apportée sous forme de $\mathrm{Fe}$ EDTA, n'ont eu aucun effet, l'apport du fer sous forme de Séquestrène (Fe 138), chélate du commerce, non seulement corrige les symptômes mais améliore la croissance quand il est utilisé à la dose de $200 \mathrm{mg} / \mathrm{l}$ (fig. 2).

Le milieu de multiplication permet, sans modification, d'obtenir des pousses feuillées assez allongées pour aborder l'enracinement.

\section{Enracinement}

Les tentatives ont été réalisées à partir de pousses feuillées de 3 à $5 \mathrm{~cm}$ de longueur.

Trois auxines ont été associées au milieu de multiplication dépourvu de cytokinine et d'acide gibbérellique : l'ANA et l'AIA ne donnent aucun résultat mais l'AIB à la concentration de $0,1 \mathrm{mg} / \mathrm{l}$ entraîne une production de racines d'aspect normal (fig. 3), les doses supérieures provoquant soit la formation de cal associé à des racines charnues $(0,5 \mathrm{mg} / \mathrm{l})$, soit seulement de cal $(1 \mathrm{mg} / \mathrm{l})$. Plusieurs essais réalisés chacun avec 24 pousses feuillées ont permis de noter un succès dans 85 à 90 p. 100 des cas.

L'apport de fer sous forme de Séquestrène Fe 138 favorise la croissance des racines.

\section{B. Matériel adulte}

Sur tous les milieux utilisés (tabl. 1), nous avons observé un brunissement général des explantats quand

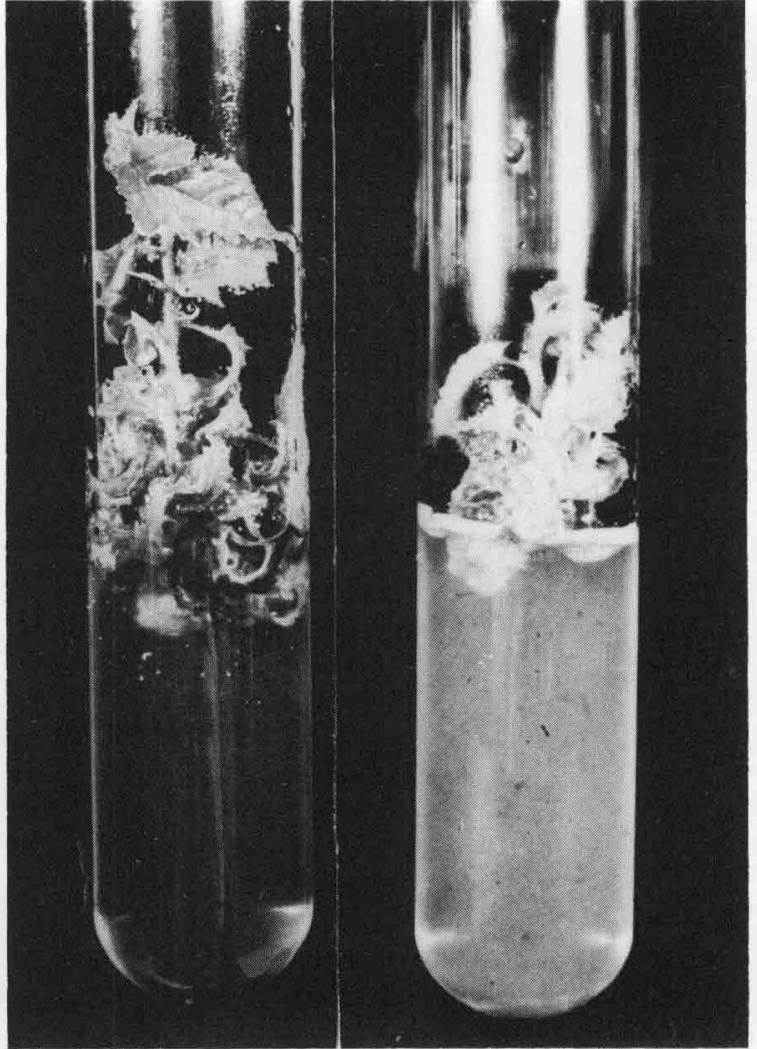

Figure 2

Effet de la nature de l'apport en fer.

A gauche, avec séquestrène: feuilles vertes, bonne croissance. A droite, avec $\mathrm{Fe}$ (EDTA) : feuilles chlorotiques, faible croissance.

Effect of the type of iron supply.

Left, with sequestrene: green leaves, good growth.

Right, with Fe (EDTA): chlorotic leaves, weak growth.

le matériel a pour origine des pieds-mères pour marcottes. Il est moindre, d'une part, quand les prélèvements ont lieu en serre sur marcotte de 2 ans et, d'autre part, quand la solution minérale de MuRAsHIGE \& SKOOG est diluée de moitié.

Deux à 3 passages sur ce milieu optimum $(\mathrm{Ms} / 2+$ BAP $(5 \mathrm{mg} / \mathrm{l})$ ne conduisent qu'à une multiplication faible.

Par contre, le transfert sur le milieu de multiplication défini pour le matériel juvénile améliore sensiblement les résultats.

Il paraît donc souhaitable, après avoir obtenu le démarrage des axillaires en présence d'une solution minérale (MS) diluée de moitié, de transférer ceux-ci sur le milieu suivant : solution minérale $\mathrm{Ms}$, mélange vitaminique de ZUCCHERELLI, AG $(0,1 \mathrm{mg} / \mathrm{l})$, ANA $(0,01 \mathrm{mg} / \mathrm{l})$, BA $(5 \mathrm{mg} / 1)$.

Si cette formule s'est avérée la meilleure, le taux de multiplication reste nettement inférieur à celui qui est noté avec le matériel juvénile.

Allongement et enracinement sont obtenus dans les mêmes conditions que pour ce dernier matériel. Aucun problème particulier n'est apparu lors du transfert en serre.

\section{DISCUSSION ET CONCLUSION}

$\mathrm{Au}$ cours de la mise au point de ces techniques, nous nous sommes heurtés à un problème inhabituel 


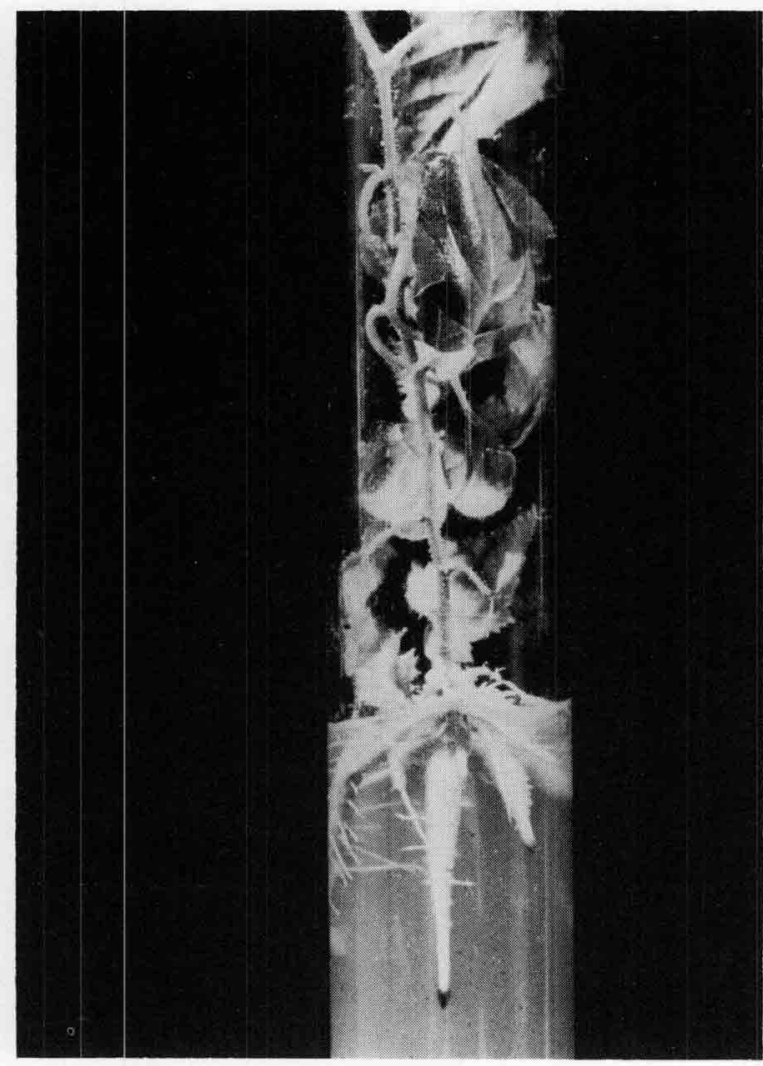

a

Figure 3

Phase d'enracinement

a) racines anormales obtenues avec $0,5 \mathrm{mg} / \mathrm{l} \mathrm{d}^{\prime} \mathrm{AIB}$

b) racines normales obtenues avec $0,1 \mathrm{mg} / \mathrm{l} d^{\prime} A I B$.

avec les autres espèces fruitières : la « décoloration » des limbes foliaires. Il a pu être résolu par l'apport de fer sous une forme (Fe EDDHA) peu utilisée en culture in vitro; d'après les effets observés au cours des divers essais, il est vraissemblable que ce chélate, qui certes apporte le fer, a aussi une autre action qui s'exerce en particulier sur la croissance : cet effet a déjà été souligné par les agronomes il y a de nombreuses années (HEATH \& CLARK, 1956).

Si la BAP s'avère indispensable au déclenchement de la multiplication, comme pour la plupart des espèces ligneuses (Prunus : SEIRLIS et al., 1979 ; Casta-
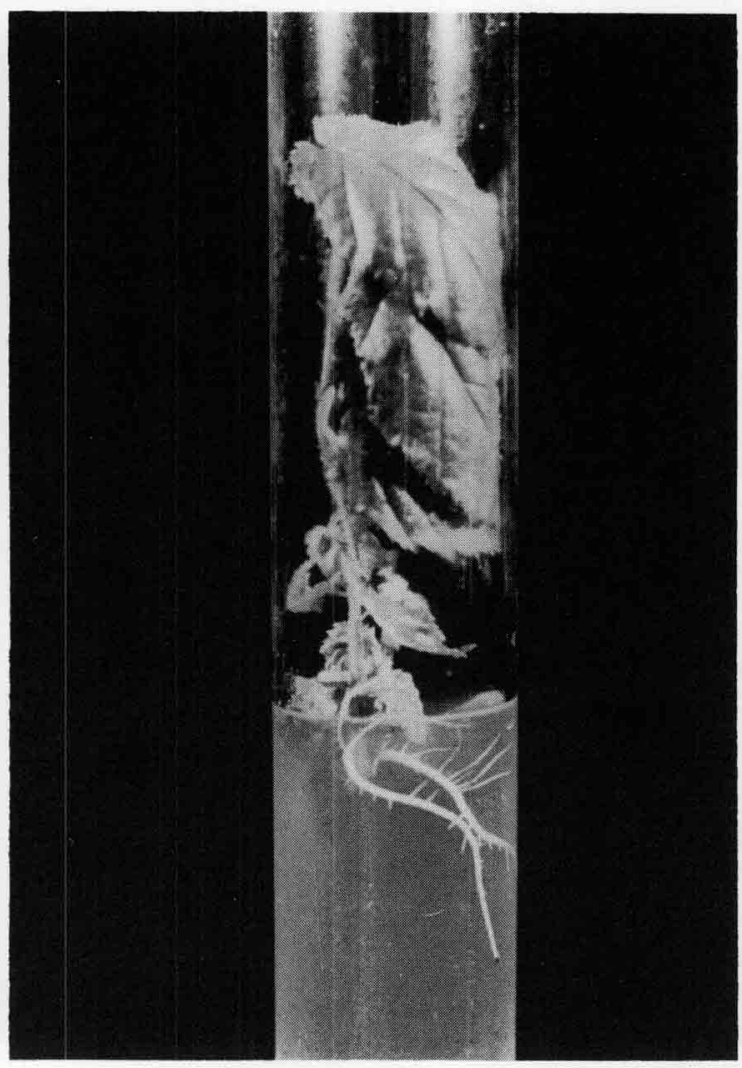

b

Rooting stage

a) anomalous roots obtained with $0.5 \mathrm{mg} / \mathrm{l} \mathrm{IBA}$

b) normal roots obtained with $0.1 \mathrm{mg} / \mathrm{l} I B A$.

nea : CHEVRE et al., 1983), c'est seulement grâce à l'utilisation de ce chélate de fer que nous avons pu progresser dans le cas du noisetier.

Les résultats obtenus sur matériel juvénile et adulte montrent qu'il doit être possible de parvenir à la mise au point d'une technique de micropropagation intéressante chez cette espèce. Une amélioration doit cependant être apportée en ce qui concerne le matériel adulte pour envisager une multiplication à grande échelle.

Reçu le 26 mai 1983. Accepté le 3 janvier 1984.

\section{RÉFÉRENCES BIBLIOGRAPHIQUES}

Chèvre Anne-Marie, Gill S. S., Mouras A., Salesses G., 1983. In vitro vegetative multiplication of chestnut. J. Hortic. Sci., $\mathbf{5 8}$ (1), 23-29.

Heath O. Y. S., Clark J. E., 1956. Chelating agents as plant growth substances. A possible clue to the mode of action of auxin. Nature, 177, 1118-1121.

Murashige T., Skoog F., 1962. A revised medium for rapid growth and bioassays with tobacco tissue culture. Physiol. Plant., 18, 100-127.

Perez C., Rodriguez R., Sanchez Taures R., 1982. Influence of germination on callus initiation and root formation from in vitro culture of filbert cotyledons. Abstr. 79th Annu. Meet. Am. Soc. Hortic. Sci., IOWA State University. August 8-13, 1982. Hortic. Sci., 17 (3) 102.

Quoirin M., Lepoivre Ph., Boxus Ph., 1976-1977. Un premier bilan de dix années de recherches sur les cultures de méristèmes et la multiplication in vitro de fruitiers ligneux. Extr. C.-R. Rech. 1976-1977, Rapp. Synth., Stn. Cult. fruitières et maraîchères, Cent. Rech. agron. de l'Etat, Gembloux (Belgique), 93-117.

Radojevic Ljiljana, 1980. Embryogenèse somatique et androgenèse chez certaines espèces ligneuses. Bull. Soc. Bot. Fr., 127, Actual. Bot. (314), 99-107.

Seirlis G., Mouras A., Salesses G., 1979. Tentative de culture in vitro d'anthères et de fragments d'organes chez les Prunus. Ann. Amélior. Plant., 29 (2), 145-161.

Zuccherelli G., 1979. Metodologie nella moltiplicazione industriale in vitro dei portainnesti donali del Pesco : «Pescamandorlo GF 677 » «Susino GF 43 » «Damasco $1869 » 《 S$. Giuliano $655 / 2$ ». Incontro su «Techniche di colture in vitro per la propagazione su vasta scala delle specie ortoflorofrutticole », Pistoia, 6 ottobre $1979,147-154$. 